Proyecciones Journal of Mathematics

Vol. 37, No 4, pp. 699-711, December 2018.

Universidad Católica del Norte

Antofagasta - Chile

\title{
Multiset ideal topological spaces and local functions
}

\author{
Karishma Shravan \\ Institute of Adv. Study in Sc. and Tech., India \\ and \\ Bimal Chandra Tripathy \\ Tripura University, India \\ Received : February 2018. Accepted : March 2018
}

\begin{abstract}
In this article we have introduced the notion of multiset local function on an ideal topological space using the the concept of q-neighbourhood in a multiset topological space. Some basic properties of local functions on multisets have been investigated in multiset ideal topological space.
\end{abstract}

Mathematics Subject Classification. 11T23; 20G40; 54A05; 54A10; $94 B 05$.

Keywords. Multiset; Local Function; Ideal; Quasi-coincidence. 


\section{Introduction}

Multisets, like sets, are collection of elements but with the underlying assumption that its elements can be repeated. The number of times an element repeats is called its multiplicity. In the real world, one can speculate enormous repetition. For instance, there are many hydrogen atoms, repeated statistical datas, many stands of DNA etc. This has motivated many researcher to develop the theory of multisets which claims to have three possible relations among any two physical entity; they are different, they are same but separate, or they are coinciding and identical.

The term Multiset was first coined by N. G. deBruijn stating it as a generalization of a set. Besides its applications in the field computer science, physics, logic, many fields of mathematics have been explored in context of multisets. The topological structures of multisets have been introduced by Girish et.al. [6]. They also studied in detail some basic notions and properties like basis, limit points, closure, interior and continuity on multiset topological space Girish et.al. [7]. After the introduction of a topology on multisets many researcher have put their effort on studying many topological properties of multisets $[4,5,6,11]$.

The notion of ideal topology was introduced by Kuratowski [10]. There after Vaidyanathaswamy [20] studied the concept of ideals in point set topology. It was followed by the investigations on ideal topological and ideal fuzzy topological spaces by Tripathy and Acharjee [13], Triapthy and Ray [19]. Jankovic and Hamlett [8] investigated further properties of ideal topological spaces where they studied that ideal is a generalization, or unification, of the concept of closure point, $w$-accumulation point, condensation point and the point of second category. Also, on considering the ideals of subsets on $N$, the set of natural numbers, the notion of $I$-convergent sequences have been studied by Tripathy and Dutta [14], Tripathy and Hazarika ([15], [16], [17]), Tripathy and Mahanta [18] and others. The concept of local function of an ideal in general topology was further introduced by Kuratowski [10]. Zakaria et al [21] have given a brief introduction to ideals in a multiset topological space.

In this article our aim is to study the properties of local function of multiset ideals in multiset topological space. 


\section{Definitions and Preliminaries}

In this section we procure some basic definitions, results, and notations to be used in this article.

Definition 2.1. A domain $X$, is defined as the set of elements from which msets are constructed. The mset space $[X]^{w}$ is the set of all msets whose are from $X$ such that no element occurs more than $w$ times.

Throughout this paper, we denote a multiset drawn from the multiset space $[X]^{w}$ by $M$.

Definition 2.2. A mset $M$ drawn from the set $X$ is represented by a function count $M$ or $C_{M}: X \rightarrow N$ where $N$ represents the set of non-negative integers.

Here $C_{M}(x)$ is the number of occurrences of the element $x$ in the mset $M$ drawn from the set $X=\left\{x_{1}, x_{2}, \ldots \ldots, x_{n}\right\}$ as $M=\left\{m_{1} / x_{1}, m_{2} / x_{2}, \ldots \ldots . m_{n} / x_{n}\right\}$ where $m_{i}$ is the number of occurrences of the element $x_{i}, i=1,2, \ldots . n$ in the mset $M$. The elements which are not included in the mset $M$ have zero count, by convention.

Example 2.1. Let $X=\{p, q, r, s\}$ be any set. Then $\{3 / p, 4 / q, 2 / r, 5 / s\}$ is an mset drawn from $X$. Clearly a set is a particular case of an mset, in which every element has multilpicity 1 .

Consider two msets $M$ and $N$ drawn from a set $X$. The following are the operations defined on the msets will be used in the article

1. $M=N$ if $C_{M}(x)=C_{N}(x)$ for all $x \in X$.

2. $M \subseteq N$ if $C_{M}(x)=C_{N}(x)$ for all $x \in X$.

3. $P=M \cup N$ if $C_{P}(x)=\max \left\{C_{M}(x), C_{N}(x)\right\}$ for all $x \in X$.

4. $P=M \cap N$ if $C_{P}(x)=\min \left\{C_{M}(x), C_{N}(x)\right\}$.

5. $P=M \oplus N$ if $C_{P}(x)=C_{M}(x)+C_{N}(x)$ for all $x \in X$.

6. $M^{c}=Z-M=\left\{C_{M^{c}}(x) / x: C_{M^{c}}(x)=C_{Z}(x)-C_{M}(x) ; x \in X\right\}, Z$ be a mset with maximum multiplicity in the multiset space. 
7. $P=M \ominus N$ if $C_{P}(x)=\max \left\{C_{M}(x)-C_{N}(x), 0\right\}$ for all $x \in X$,

where $\oplus$ and $\ominus$ represent mset addition and mset subtraction respectively.

\subsection{Operations on collection of msets}

Let $[X]^{w}$ be an mset space and $Z$ be a subset of $[X]^{w}$ with $C_{Z}(x)$ as the multiplicities of $x \in X$ in $Z$. Let $\left\{M_{1}, M_{2}, \ldots ..\right\}$ be a collection of msets drawn from $[X]^{w}$. Then the following operations are possible under on an arbitrary collection $\left\{M_{i}: i \in \Delta\right\}$ of msets, where $\Delta=\{1,2, \ldots\}$ is the support set.

1. The Union

$\prod_{i \in \Delta} M_{i}=\left\{C_{M_{i}}(x) / x: C_{M_{i}}(x)=\max \left\{C_{M_{i}}(x): x \in X\right\}\right\}$.

2. The intersection

$\cap_{i \in \Delta} M_{i}=\left\{C_{\cap M_{i}}(x) / x: C_{\cap M_{i}}(x)=\min \left\{C_{M_{i}}(x): x \in X\right\}\right.$.

3. The mset complement $M^{c}=Z \ominus M=\left\{C_{M^{c}}(x) / x: C_{M^{c}}(x)=C_{Z}(x)-C_{M}(x), x \in X\right\}$.

Definition 2.3. Let $M$ be an mset drawn from a set $X$. The Support set of $M$ denoted by $M^{*}$ is a subset of $X$ and $M^{*}=\left\{x \in X: C_{M}(x)>0\right\}$.

Definition 2.4. An mset $M$ is said to be an empty set if for all $x \in X$, $C_{M}(x)=0$.

Definition 2.5. Let $X$ be a support set and $[X]^{w}$ be the mset space defined over $X$. Then for any mset $M \in[X]^{w}$, the complement $M^{c}$ of $M$ in $[X]^{w}$ is an element of $[X]^{w}$ such that $C_{M^{c}}=w-C_{M}(x)$ for all $x \in X$.

Since Cantor's power set theorem fails for msets, it is possible to formulate the following reasonable definition of a power mset of $M$ for finite mset $M$ that preserves Cantor's power set theorem.

Definition 2.6. Let $M \in[X]^{w}$ be an mset. The power mset $P(M)$ of $M$ is the set of all submsets of $M$. We have $N \in P(M)$ if and only if $N \subseteq M$. If $N=\emptyset$, then $N \in^{1} P(M)$ and if $N \neq \emptyset$, then $N \in^{k} P(M)$, where $k=\prod_{z}\left(\begin{array}{c}{[M]_{z}} \\ {[N]_{z}}\end{array}\right)$, the product $\prod_{z}$ is taken over by distinct elements $z$ 
of the mset $N$ and $\left|[M]_{z}\right|=m$ if and only if $z \in^{m} M$ and $\left|[N]_{z}\right|=n$ if and only if $z \in^{n} N$ then,

$$
\left(\begin{array}{l}
{[M]_{z}} \\
{[N]_{z}}
\end{array}\right)=\left(\begin{array}{c}
m \\
n
\end{array}\right)=\frac{m !}{n !(m-n) !} .
$$

The power set of an mset is the support set of the power mset and is denoted by $P^{*}(M)$.

Note 2.1. Power mset is an mset but its support set is an ordinary set whose elements are msets.

\subsection{Multiset Topology}

Girish and John [6] introduced the concept of Multiset topology and defined as follows:

Let $M \in[X]^{w}$ and $\tau \subseteq P^{*}(M)$. Then $\tau$ is called a multiset topology of $M$ if $\tau$ satisfies the following properties.

1. The mset $M$ and the empty mset $\emptyset$ are in $\tau$.

2. The mset union of elements of any subcollection of $\tau$ is in $\tau$.

3. The mset intersection of the elements of any finite subcollection of $\tau$ is in $\tau$.

Mathematically a multiset topological space is an ordered pair $(M, \tau)$ consisting of an mset $M$ extracted from $[X]^{w}$ and a multiset topology $\tau \subseteq P^{*}(M)$ on $M$. Multiset topology is abbreviated as $M$-Topology. The elements of $\tau$ are called open mset. The complement of an open mset in a $M$-Topological space is said to be closed mset.

Definition 2.7. Given a submset $A$ of $M$-topological space $M$ in $[X]^{w}$, the interior of $A$ is denoted by $\operatorname{int}(A)$ and is defined as the mset union of all opem msets contained in $A$.

$$
\text { i.e } C_{\text {int }(A)}(x)=\max \left\{C_{G}(x): G \subseteq A\right\} \text {. }
$$


Definition 2.8. Given a submset $A$ of an $M$-topological space $M$ in $[X]^{w}$, the closure of $A$ is defined by the mset intersection of all closed msets containing $A$ and is denoted by $\operatorname{cl}(A)$, i.e $C_{C l(A)}(x)=\min \left\{C_{K}(x): A \subseteq K\right\}$.

The notion of ideal is defined as follows,

Definition 2.9. A nonempty collection of subsets $I$ of a nonempty set $X$ is called a ideal on $X$ if the following postulates are satisfied

(i) if $A \in I$ and $B \subseteq A$, then $B \in I$ (heredity),

(ii) if $A, B \in I$, then $A \cup B \in I$ (additivity).

The triplet $(X, \tau, I)$ is called ideal fuzzy topological space with the ideal $I$ and fuzzy topology $\tau$.

The notion of mset ideal is defined in Zakaria et.al.[21] as follows,

Definition 2.10. A non-empty collection $I$ of submsets of a non-empty mset $M$ is said to be an mset ideal on $M$, if it satisfies the following conditions:

1. $N_{1} \in I$ and $C_{N_{2}}(x) \leq C_{N_{1}}(x)$ for all $x \in X \Rightarrow N_{2} \in I$.

2. $N_{1} \in I, N_{2} \in I \Rightarrow N_{1} \cup N_{2} \in I$.

The mset ideal is abbreviated as $M$-ideal.

Let $M$ be an infinite set. Then consider the family of subsets by $M$, defined by $I=\{N \subseteq M: N$ is finite $\}$. It can be easily verified that $I$ is a mset ideal of $M$. For further examples of mset ideals, one may refer to Zakaria et.al.[21].

It is proved by Zakaria et.al.[21] that the union and intersection of two mset ideals is a mset ideal.

\section{Main Results}

Definition 3.1. Let $[X]^{w}$ be a space of multisets. A multipoint is a multiset $M$ in $X$ such that 


$$
\begin{gathered}
C_{M}(x)=k, \text { for } x \in M ; \\
=0, \text { otherwise }
\end{gathered}
$$

where $k \varepsilon\{1,2,3, \ldots \ldots . w\}$ and $C_{M}(x)$ is the multiplicity of $x$ in $X$.

Note 3.1. A multipoint, denoted by $\{k / x\}$ is a subset of a multiset $M$ or $\{k / x\} \in M$ if $k \leq C_{M}(x)$.

\subsection{Quasi-coincidence in Multisets}

Definition 3.2. Let $M$ be any multiset in the space $[X]^{w}$. If $N \subseteq M$, then $k / x \in M$ is said to be quasi-coincident with $N$ if and only if $k>C_{N^{c}}(x)$.

Definition 3.3. A multiset $M$ is said to be quasi-coincident with $N$ i.e. $M q N$ at $x$ if and only if $C_{M}(x)>C_{N^{c}}$.

Remark 3.1. It can be easily verified, if $M$ and $N$ are quasi-coincident at $x$ then both $C_{M}(x)$ and $C_{N}(x)$ are non-zero and hence $M$ and $N$ intersect at $x$.

Definition 3.4. A multiset $N$ in an $M$-Topological space $(M, \tau)$ is said to be a $q$-neighborhood( $Q$-nbhd) of $k / x$ if and only if there exists an open mset $P$ such that,

$$
k / x q P \subset N
$$

\subsection{Multiset Local Function}

Let $(M, \tau)$ be a $M$-topological space and $\mathcal{I}$ be an $M$-ideal on $M$. If $N$ is any submset of $M$, then the local function denoted by $N^{*}(I, \tau)$ is defined by,

$$
\begin{aligned}
& N^{*}(\mathcal{I}, \tau) \\
&= \cup\left\{m_{i} / x_{i} \in M: C_{U}\left(x_{j}\right)-C_{N^{c}}\left(x_{j}\right)>C_{I}\left(x_{j}\right), I \in \mathcal{I} \text { for every } U \in\right. \\
& N_{q}\left(m_{i} / x_{i}\right)
\end{aligned}
$$

and at least one $\left.x_{j} \in X\right\}$,

where $N_{q}\left(m_{i} / x_{i}\right)$ is the set of $q$-nbhd of $m_{i} / x_{i}$. 
We will write $N^{*}(\mathcal{I})$ or $N^{*}$ in place of $N^{*}(\mathcal{I}, \tau)$.

Lemma 3.1. Show that $N^{*}(\emptyset)=C l N$.

Proof. On putting $\mathcal{I}=\emptyset$ in the expression for $N^{*}(I)$, we have by definition of closure in $M$-topology,

$N^{*}(\emptyset)=\cup\left\{m_{i} / x_{i} \in M: C_{U}\left(x_{j}\right)-C_{N^{c}}\left(x_{j}\right)>0, I \in \mathcal{I}\right.$, for all $U \in$ $N_{q}\left(m_{i} / x_{i}\right)$, and at least one $\left.x_{j} \in X\right\}$

$=\cup\left\{m_{i} / x_{i} \in M: C_{U}\left(x_{j}\right)>C_{N^{c}}\left(x_{j}\right), I \in \mathcal{I}\right.$, for all $U \in N_{q}\left(m_{i} / x_{i}\right)$, and at least one $\left.x_{j} \in X\right\}$

$=C l(N)$.

Example 3.2. $N^{*}\left(P^{*}(M)\right)=\emptyset$.

It is obvious, as for every $m_{i} / x_{i} \in M$ there is at least one $U \in N_{q}\left(m_{i} / x_{i}\right)$ such that for every $x_{j} \in X, C_{U}\left(x_{j}\right)-C_{N^{c}}\left(x_{j}\right) \leq C_{I}\left(x_{j}\right), I \in P^{*}(M)$.

\subsection{Properties of multiset local function}

Throughout this section $(M, \tau)$ will denote a $M$-topological space with $\mathcal{I}$ and $\mathcal{J}$ as two $M$-ideals on $M$.

Theorem 3.1. If $N, P \subseteq M$ then, $N \subseteq P \Rightarrow N^{*}(\mathcal{I}, \tau) \subseteq P^{*}(\mathcal{I}, \tau)$.

Proof. By definition of local function, we have

$N^{*}(\mathcal{I})=\cup\left\{m_{i} / x_{i} \in M: C_{U}\left(x_{j}\right)-C_{N^{c}}\left(x_{j}\right)>C_{I}\left(x_{j}\right), I \in \mathcal{I}\right.$ for every $U \in N_{q}\left(m_{i} / x_{i}\right)$ and at least one $\left.x_{j} \in X\right\}$.

$P^{*}(\mathcal{I})=\cup\left\{m_{i} / x_{i} \in M: C_{U}\left(x_{j}\right)-C_{P^{c}}\left(x_{j}\right)>C_{I}\left(x_{j}\right), I \in \mathcal{I}\right.$ for every $U \in N_{q}\left(m_{i} / x_{i}\right)$ and at least one $\left.x_{j} \in X\right\}$,

where $N_{q}\left(m_{i} / x_{i}\right)$ is the set of $q-n b h d$ of $m_{i} / x_{i}$.

Since,

$N \subseteq P$

$\Rightarrow C_{N}\left(x_{j}\right) \leq C_{P}\left(x_{j}\right) \forall x_{j} \in X$

$\Rightarrow C_{P^{c}}\left(x_{j}\right) \leq C_{N^{c}}\left(x_{j}\right)$ for all $x_{j} \in X$

$\Rightarrow C_{U}\left(x_{j}\right)-C_{N^{c}}\left(x_{j}\right) \leq C_{U}\left(x_{j}\right)-C_{P^{c}}\left(x_{j}\right)$. 
Hence, $N^{*}(\mathcal{I}, \tau) \subseteq P^{*}(\mathcal{I}, \tau)$.

Theorem 3.2. If $\mathcal{I} \subseteq \mathcal{J} \Rightarrow N^{*}(\mathcal{J}, \tau) \subseteq N^{*}(\mathcal{I}, \tau)$.

Proof. We have,

$N^{*}(\mathcal{I})=\cup\left\{m_{i} / x_{i} \in M: C_{U}\left(x_{j}\right)-C_{N^{c}}\left(x_{j}\right)>C_{I}\left(x_{j}\right), I \in \mathcal{I}\right.$ for every $U \in N_{q}\left(m_{i} / x_{i}\right)$ and at least one $\left.x_{j} \in X\right\}$.

$N^{*}(\mathcal{J})=\cup\left\{m_{i} / x_{i} \in M: C_{U}\left(x_{j}\right)-C_{N^{c}}\left(x_{j}\right)>C_{J}\left(x_{j}\right), J \in \mathcal{J}\right.$ for every $U \in N_{q}\left(m_{i} / x_{i}\right)$ and at least one $\left.x_{j} \in X\right\}$.

Since, $\mathcal{I} \subseteq \mathcal{J}$, then there exist msets in $\mathcal{J}$ so that for any $m$-point $m_{i} / x_{i}, m_{i} / x_{i} \in N^{*}(\mathcal{I})$ but $m_{i} / x_{i}$ may not belong to $N^{*}(\mathcal{J}, \tau)$.

Hence, $N^{*}(\mathcal{J}, \tau) \subseteq N^{*}(\mathcal{I}, \tau)$.

Theorem 3.3. $(N \cup P)^{*}(\mathcal{I}, \tau)=N^{*}(\mathcal{I}, \tau) \cup P^{*}(\mathcal{I}, \tau)$.

Proof. Let $m / x \notin N^{*} \cup P^{*}$. Then,

$m>C_{N^{*} \cup P^{*}}(x)=\max \left\{C_{N^{*}}(x), C_{P^{*}}(x)\right\}$ for all $x \in X$, i.e $m / x \notin N^{*}$ and $m / x \notin P^{*}$.

So, for every $y \in X$ there exists at least one $q$-nbhd $U$ of $m / x$ such that

$C_{V}(y)-C_{P}^{c}(y) \leq C_{J}(y)$ for some $J \in \mathcal{J}$.

Also, for every $y \in X$, there exists at least one $q$-nbhd $V$ of $m / x$ such that

$C_{U}(y)-C_{N}^{c}(y) \leq C_{I}(y)$ for some $I \in \mathcal{I}$.

Taking, $C_{W}(y)=\min \left\{C_{U}(y), C_{V}(y)\right\}$, we have

$C_{W}(y)-C_{N^{c} \cap P^{c}}(y) \leq C_{I \cup J}(y), I \cup J \in \mathcal{I}$, i.e. $\left.C_{W}(y)-C_{(} N \cup P\right)^{c} \leq$ $C_{I \cup J}(y)$.

So, $m / x \notin(N \cup P)^{*}$ and hence $(N \cup P)^{*} \subseteq N^{*} \cup P^{*}$. 
Clearly, $N^{*} \cup P^{*} \subseteq(N \cup P)^{*}$.

Therefore, $(N \cup P)^{*}(\mathcal{I}, \tau)=N^{*}(\mathcal{I}, \tau) \cup P^{*}(\mathcal{I}, \tau)$.

Theorem 3.4. $N^{*}=C l\left(N^{*}\right) \subseteq C l(N)$.

Proof. $N^{*}(\mathcal{I})=\cup\left\{m_{i} / x_{i} \in M: C_{U}\left(x_{j}\right)-C_{N^{c}}\left(x_{j}\right)>C_{I}\left(x_{j}\right), I \in \mathcal{I}\right.$ for every $U \in N_{q}\left(m_{i} / x_{i}\right)$ and at least one $\left.x_{j} \in X\right\}$.

We have for any $M$-ideal $\mathcal{I},\{\emptyset\} \subseteq \mathcal{I}$. So, by Definition 3.1 we have $N^{*}(\mathcal{I}) \subseteq N^{*}(\emptyset)=C l N$.

Therefore,

$$
N^{*}(\mathcal{I}) \subseteq C l N
$$

Let $m_{1} / x_{1} \in C l\left(N^{*}\right)$.

So, there exists a $q$-nbhd $U$ of $m_{1} / x_{1}$ such that $U q N^{*}$ i.e $C_{U}(y)>C_{\left(N^{*}\right)^{c}}$ $\forall y \in X$.

Hence $C_{N^{*}}(y) \neq 0$ and $C_{N}^{*}(y)=m_{2}$ (say).

i.e $m_{2} / y \in N^{*}$. So there exists a $q$-nbhd $V$ of $m_{2}$ such that for at least one $x_{2} \in X$,

and,

$$
C_{V}\left(x_{2}\right)-C_{N^{c}}\left(x_{2}\right)>C_{I}\left(x_{2}\right), I \in \mathcal{I}
$$

$C_{U}\left(x_{3}\right)-C_{N^{c}}\left(x_{3}\right) i C_{I}\left(x_{3}\right), I \in \mathcal{I}$ for at least one $x_{3} \in X$

Since, $U$ is a $q$-nbhd of $m_{1} / x_{1}, m_{1} / x_{1} \in N^{*}$.

Therefore, $C l\left(N^{*}\right) \subseteq N^{*}$.

So, $C l\left(N^{*}\right)=N^{*} \subseteq C l N$.

Theorem 3.5. $\left(N^{*}\right)^{*} \subseteq N^{*}$.

Proof. By (1), we have $\left(N^{*}\right)^{*}=C l\left(\left(N^{*}\right)^{*}\right) \subseteq C l\left(N^{*}\right)=N^{*}$. 
Theorem 3.6. $I \in \mathcal{I} \Rightarrow(N \cup I)^{*}=N^{*}$.

Proof. It is obvious that for $I \in \mathcal{I}, I^{*}=\emptyset$. Hence, by Theorem 3.2 we have,

$(N \cup I)^{*}=N^{*} \cup I^{*}=N^{*}$, by $(2)$.

\section{References}

[1] W. D. Blizard, Multiset Theory, Notre Dame Journal of Formal Logic, 30 (1), pp. 36-66, (1989).

[2] W. D. Blizard, The development of multiset theory, Modern Logic, 1 (4), pp. 319-352, (1991).

[3] S. El-Sheikh, R. Omar, M. Raafat, Separation Axioms on Multiset Topology, Journal of New Theory, 7, pp. 11-21, (2015).

[4] S. El-Sheikh, Rajab Omar, Mahmoud Raafat, Separation Axioms on Multiset Topology, Journal of New Theory, 7, pp. 11-21, (2015).

[5] S. A. El-Sheikh, R. A-K. Omar, and, M. Raafat, Operators on multiset bitopological spaces, South Asian Jour. Math., 6 (1), pp. 1-9, (2016).

[6] K. P. Girish and S. J. John, Rough multiset and its multiset topology In: Transactions on Rough Sets XIV, Springer; Berlin, Heidelberg. Vol. 6600, Lecture Notes in Computer Science, pp. 62-80, (2011).

[7] K. P. Girish and S. J. John, On Multiset Topologies, Theory Appl. Math. Comput. Sci., 2, pp. 37-52, (2012).

[8] D. Jankovic and T. R. Hamlen, New topologies from old via ideals, Amer. Math. Monthly 97, pp. 295-310, (1990).

[9] A. Kandia, O. A. Tantawy, S. A. El-Sheikh, A. Zakaria, Multiset proximity spaces, Jour. Egyptian Math. Soc., 24, pp. 562-567, (2016).

[10] K. Kuratowski, Topologie I, Warszawa, (1933).

[11] P. M. Mahalakshmi, P. Thangavelu, $M$-Connectedness in $M$-Topology, Internat. Jour. Pure Appl. Math., 106 (8), pp. 21-25, (2016). 
[12] K. Shravan and B. C. Tripathy, Generalized closed sets in multiset topological space, Proyecciones J. Math., (Accepted for Publication).

[13] B. C. Tripathy and S. Acharjee, On $(\gamma, \delta)$-Bitopological semi-closed set via topological ideal, Proyecciones J. Math., 33 (3), pp. 245-257, (2014).

[14] B. C. Tripathy and A. J. Dutta, Lacunary I-convergent sequences of fuzzy real numbers, Proyecciones J. Math., 34 (3), pp. 205-218, (2015).

[15] B. C. Tripathy and B. Hazarika, I-convergent sequence spaces associated with multiplier sequence spaces, Math. Ineq. Appl., 11 (3), pp. 543-548, (2008).

[16] B. C. Tripathy and B. Hazarika, I-monotonic and $I$-convergent sequences, Kyungpook Math. J., 51 (2), pp. 233-239, (2011).

[17] B. C. Tripathy; B. Hazarika and B. Choudhary, Lacunary I-convergent sequences, Kyungpook Math. J., 52 (4), pp. 473-482, (2012).

[18] B. C. Tripathy and S. Mahanta, On I-acceleration convergence of sequences, Jour. Franklin Inst., 347, pp. 591-598, (2010).

[19] B. C. Tripathy and G. C. Ray, Mixed fuzzy ideal topological spaces, Appl. Math. Comput., 220, pp. 602-607, (2013).

[20] Vaidyanathaswamy, R., Set Topology, (Chelsea, New York), (1960).

[21] A. Zakaria, S. J. John and S. A. El-Sheikh, Generalized rough multiset via multiset ideals, Jour. Intel. Fuzzy Syst., 30, pp. 1791-1802, (2016).

\section{Karishma Shravan}

Mathematical Sciences Division

Institute of Advanced Study in Science and Technology

Guwahati - 781035; Assam;

India

e-mail: karishma_math@rediffmail.com

and 


\section{Binod Chandra Tripathy}

Department of Mathematics

Tripura University

Agartala - 799022; Tripura;

India

e-mail: binodtripathy@tripurauniv.in 